\title{
Hydrogenated 1,4-Insertion of Butadiene in the Copolymerization with Propylene Using an Isospecific Zirconocene Catalyst.
}

Takeshi Ishihara, Takeshi Shiono.

\section{Supporting information}

\section{Experimental}

Materials.

Rac-dimethylsilylbis(2-methyl-4-phenylindenyl)zirconium dichloride was commercially obtained from Boulder Scientific Company and used without further purification. Propylene (commercial grade, $99.8 \%$ ) was dehydrated by passing it through a dehydration column of Moisture Filter CP17971. 1,3-Butadiene donated from Zeon Co.(>99.5\%) was dried over $\mathrm{CaH}_{2}$ and distilled before use. Hydrogen was purchased from Nippon Sanso Co.(>99.9\%) and used as received. Modified methylaluminoxane (MMAO) which was purchased from Tosoh-Finechem. Co was used without further purification. Other chemicals commercially obtained were purified according to the usual procedures.

Polymerization procedure.

Copolymerization of propylene with 1,3-butadiene was conducted in a 100-mL stainless steel autoclave by batch-wise operation at $0{ }^{\circ} \mathrm{C}$ Toluene solutions of MMAO and the catalyst, toluene as the solvent and a toluene solution of 1,3-butadiene were added to the reactor in this order. The autoclave was cooled with liquid nitrogen and then gaseous propylene, the amount of which was measured by a mass flow meter, was introduced into the reactor. In the case of hydrogen introducing condition, hydrogen was charged to the autoclave up to $0.1 \mathrm{MPa}$. Copolymerization started by setting the reactor at the polymerization temperature. The polymerization was stopped by adding an $\mathrm{HCl} /$ methanol solution after remaining monomers had been purged. The polymer was precipitated in methanol, washed with methanol several times, collected by filtration and finally dried under vacuum at $60{ }^{\circ} \mathrm{C}$. 
Analytical procedures.

${ }^{1} \mathrm{H}$ NMR and ${ }^{13} \mathrm{C}$ NMR spectra were recorded at $120{ }^{\circ} \mathrm{C}$ on a JEOL JNM-LA600 spectrometer operated at $600.05 \mathrm{MHz}$ and 150.80 MHz, respectively, in pulse Fourier-transform mode. The spectra were obtained in 1,1,2,2-tetrachloroethane- $\mathrm{d}_{2}$ using 1,1,2,2-tetrachloroethane $\left(6.0 \mathrm{ppm}\right.$ for ${ }^{1} \mathrm{H}$ $\mathrm{NMR})$ and 1,1,2,2-tetrachloroethane- $\mathrm{d}_{2}\left(74.0 \mathrm{ppm}\right.$ for $\left.{ }^{13} \mathrm{C} \mathrm{NMR}\right)$ as internal references, respectively.

Molecular weights and molecular weight distributions of the polymers were determined by a PLGPC210 equipped with PLgel $10 \mu \mathrm{m}$ MIXED-B LS at $140{ }^{\circ} \mathrm{C}$ using o-dichlorobenzene as the solvent. Molecular weights were calibrated with monodispersed polystyrene standards and converted to polypropylene molecular weights by $\mathrm{K}$ and $\alpha$ values, $1.03 \times 10^{-4}$ and 0.780 , respectively.

Differential scanning calorimetry (DSC) measurements were performed on Seiko Instruments Inc. DSC 6200, and recorded the second heating cycle from $-50^{\circ} \mathrm{C}$ to $200^{\circ} \mathrm{C}$ at a $10^{\circ} \mathrm{C} / \mathrm{min}$ rate. 
Chemical shifts of plausible copolymer structures are summarized at Table S1.

Table S1. Chemical shifts of the poly(propylene-ran-1,3-butadiene) in the ${ }^{13} \mathrm{C}$ NMR spectra

\begin{tabular}{|c|c|c|c|}
\hline Captions & Chemical shifts & Captions & Chemical shifts \\
\hline 1 & $11.0^{[\mathrm{a}]}$ & 21 & $40.0^{[\mathrm{b}]}$ \\
\hline 2 & $14.9^{[\mathrm{a}]}$ & 22 & $35.4^{[\mathrm{b}]}$ \\
\hline 3 & $40.0^{[\mathrm{a}]}$ & 23 & $32.5^{[\mathrm{b}]}$ \\
\hline 4 & $18.7^{[\mathrm{a}]}$ & 24 & $18.2^{[\mathrm{b}]}$ \\
\hline 5 & $30.6^{[\mathrm{a}]}$ & 25 & $42.2^{[\mathrm{b}]}$ \\
\hline 6 & $41.4^{[\mathrm{a}]}$ & 26 & $27.3^{[b]}$ \\
\hline 7 & $41.6^{[\mathrm{a}]}$ & 27 & $30.5^{[b]}$ \\
\hline 8 & $38.6^{[\mathrm{a}]}$ & 28 & $35.9^{[b]}$ \\
\hline 9 & $27.5^{[\mathrm{a}]}$ & 29 & $25.6^{[\mathrm{b}]}$ \\
\hline 10 & $41.8^{[\mathrm{a}]}$ & 30 & $37.8^{[\mathrm{b}]}$ \\
\hline 11 & $37.7^{[\mathrm{a}]}$ & 31 & $29.5^{[\mathrm{b}]}$ \\
\hline 12 & $142.9^{[\mathrm{a}]}$ & & \\
\hline 13 & $111.2^{[\mathrm{a}]}$ & & \\
\hline 14 & $127.8^{[\mathrm{a}]}$ & & \\
\hline 15 & $25.7^{[\mathrm{a}]}$ & & \\
\hline 16 & $43.9^{[\mathrm{a}]}$ & & \\
\hline 17 & $28.7^{[\mathrm{a}]}$ & & \\
\hline 18 & $18.8^{[\mathrm{a}]}$ & & \\
\hline 19 & $35.2^{[\mathrm{a}]}$ & & \\
\hline 20 & $25.1^{[\mathrm{a}]}$ & & \\
\hline
\end{tabular}

[a] See reference 18. [b] Calculated figures in the present paper. 


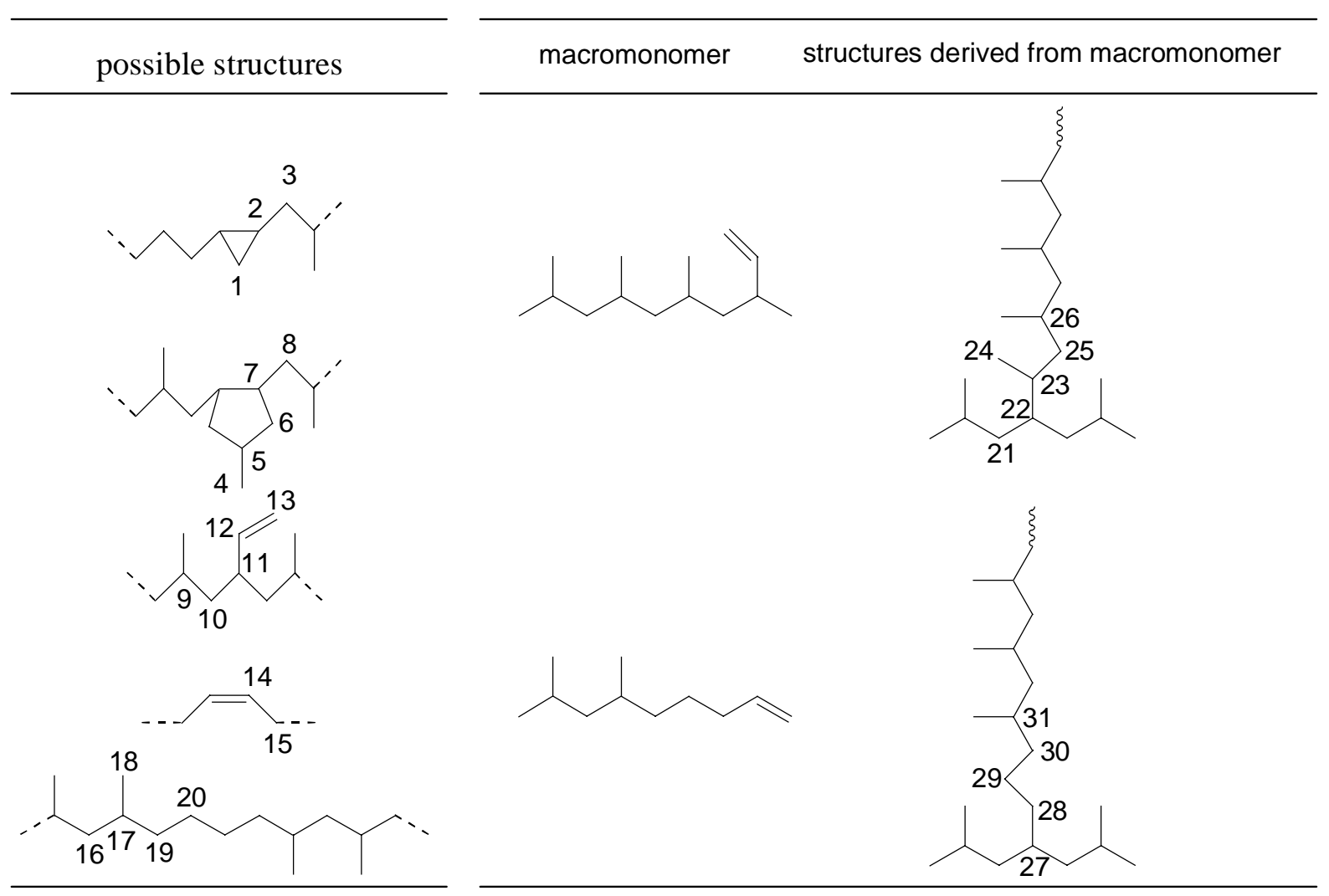




\section{Deuterium effects.}

Deuterium was used instead of hydrogen in the propylene-1,3-butadiene copolymerization in order to confirm the reaction scheme. The ${ }^{13} \mathrm{C}$ NMR spectra of the copolymers obtained with hydrogen and deuterium are shown in Figures 3 and 4. Although there is no difference between two spectra in the lower region than $40 \mathrm{ppm}$ and the higher region than $25 \mathrm{ppm}$ (Figure S1), the resonance between $25 \mathrm{ppm}$ and $38 \mathrm{ppm}$ has changed drastically in the case of using $\mathrm{D}_{2}$ (Figure S2). The resonance at $30.4_{6} \mathrm{ppm}$ (corresponding carbon atom : 1) disappeared and new several small resonances appeared at higher region.

The scheme we proposed suggests that the carbon located at $\mathbf{1}$ should have only one structure that has one deuterium. The signal attributable to $\mathbf{1}$, therefore, shifted to higher field and split into triplet because of deuterium atom. The carbon at $\mathbf{2}$ should have two structures, i.e., one directly connected with deuterium and the other connected with deuterated $\mathbf{1}$. The former appeared at higher region as a triplet and the latter shifted to higher region. The carbon at $\mathbf{3}$ should have also two kinds of structures; one located far from deuterium atom and the other connected with deuterated $\mathbf{2}$. The former kept the same chemical shit but the latter shifted to higher region. The spectrum of the copolymer obtained with $\mathrm{D}_{2}$ is in good agreement with the structure proposed in Scheme 1. 


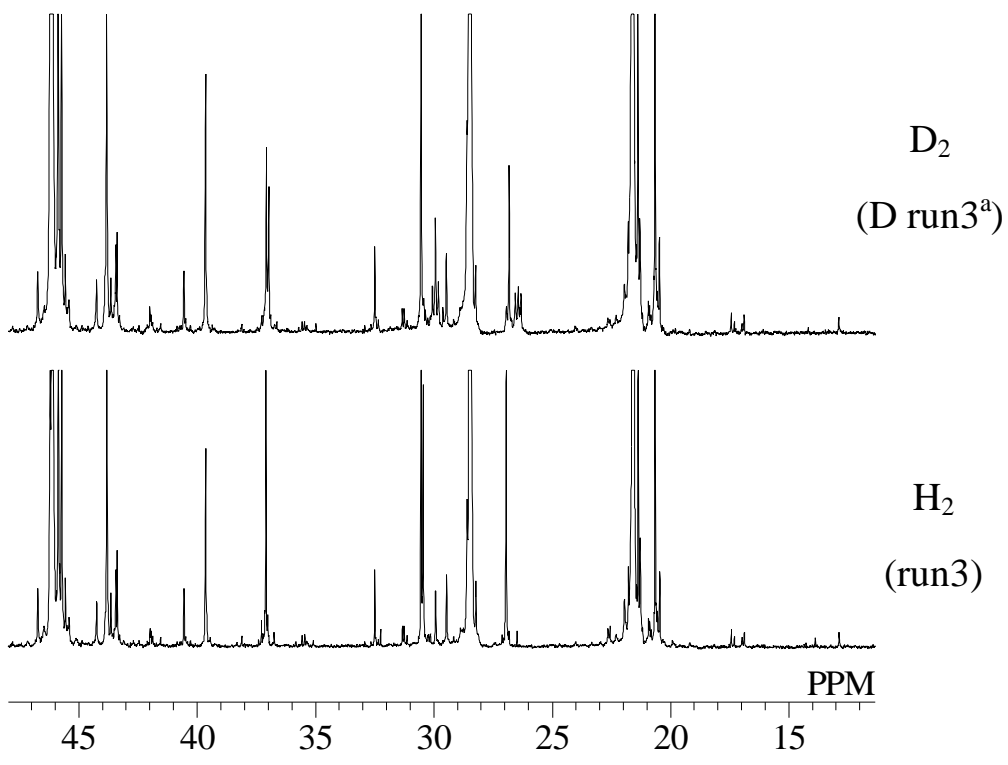

Figure S1. ${ }^{13} \mathrm{C}$ NMR spectra of poly(propylene-ran-butadiene)s obtained with hydrogen (run 3) and deuterium (D run3). ${ }^{\text {a }} \mathrm{D}$ run3 was obtained under the same conditions with run 3 using deuterium instead of hydrogen. Precise polymerization conditions, to see Table 1. 


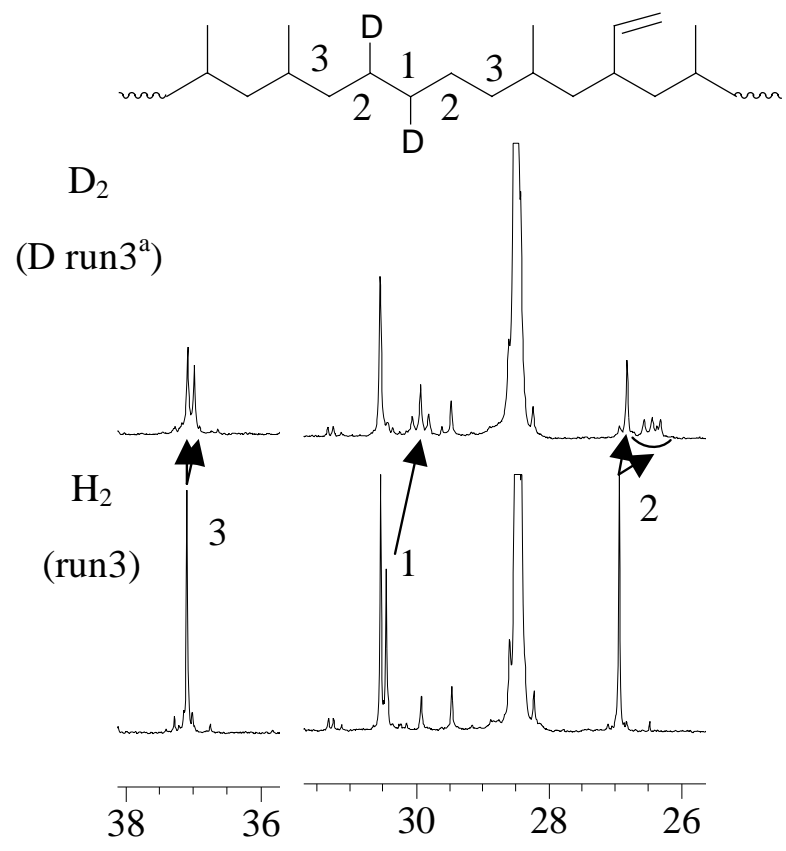

Figure S2. Expanded ${ }^{13} \mathrm{C}$ NMR spectra of poly(propylene-ran-butadiene)s obtained with hydrogen (run 3) and deuterium (D run3). ${ }^{a} D$ run3 was obtained under the same conditions with run 3 using deuterium instead of hydrogen. Precise polymerization conditions, to see Table 1. 